\title{
Overexpression of GSDMC is a prognostic factor for predicting a poor outcome in lung adenocarcinoma
}

\author{
JIE WEI ${ }^{1,2}$, ZHIJIE XU $^{3}$, XI CHEN $^{1,2}$, XIANG WANG $^{1,2}$, SHUANGSHUANG ZENG $^{1,2}$, LONG QIAN $^{1,2}$,

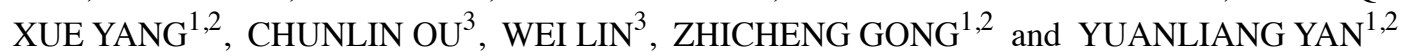 \\ ${ }^{1}$ Department of Pharmacy; ${ }^{2}$ National Clinical Research Center for Geriatric Disorders; ${ }^{3}$ Department of Pathology, \\ Xiangya Hospital, Central South University, Changsha, Hunan 410008, P.R. China
}

Received June 11, 2019; Accepted October 31, 2019

DOI: $10.3892 / \mathrm{mmr} .2019 .10837$

\begin{abstract}
The gasdermin (GSDM) superfamily has been demonstrated to consist of several important molecules that modulate multifunctional signal processes, such as cell pyroptosis. In this research, the roles of the GSDM superfamily on the occurrence and prognosis of lung adenocarcinoma (LUAD) were evaluated using integrative bioinformatic analyses and in vitro methods. Here, data from several bioinformatic platforms revealed that GSDMC is significantly upregulated in LUAD tissues and cell lines. Real-time fluorescence quantitative PCR (qPCR) demonstrated that GSDMC was obviously upregulated in radio-resistant LUAD cells, compared with their parental cells. Moreover, upregulated GSDMC expression was confirmed to be an independent indicator of poor first progression (FP) and overall survival (OS) in LUAD patients. DNA methylation analysis showed an evidently negative correlation between $G S D M C$ expression and methylation status of one CpG site (cg05316065) in its DNA sequence. Patients with high methylation values had significantly higher Karnofsky performance scores (KPSs) and prolonged OS rates. Together, we confirmed that overexpression of GSDMC acts as a promising predictive factor for the poor prognosis of LUAD patients.
\end{abstract}

\section{Introduction}

Lung cancer is the leading cause of cancer-related deaths for patients worldwide, with the highest incidence rates among all

Correspondence to: Dr Zhijie Xu, Department of Pathology, Xiangya Hospital, Central South University, 87 Xiangya Road, Changsha, Hunan 410008, P.R. China

E-mail:xzj1322007@csu.edu.cn

Dr Yuanliang Yan, Department of Pharmacy, Xiangya Hospital, Central South University, 87 Xiangya Road, Changsha, Hunan 410008, P.R. China

E-mail: yanyuanliang@csu.edu.cn

Key words: gasdermin, GSDMC, lung adenocarcinoma, prognosis, DNA methylation, genetic mutation malignancies. For the purposes of treatment, lung cancer is classified into several histological subtypes, including small cell lung cancer (13\% of cases) or non-small cell lung cancer (NSCLC, $83 \%$ of cases) $(1,2)$. In most countries, lung adenocarcinoma (LUAD) is the most frequent histological NSCLC subtype. Despite comprehensive treatment in clinical practice, most LUAD patients at present are commonly diagnosed at late stages, and meanwhile receive insufficient treatment efficacy (3). Therefore, identification of the novel biomarkers and molecular events associated with LUAD biology would provide novel insights for developing a more rational therapeutic strategy.

Pyroptosis is a form of gasdermin (GSDM)-mediated programmed necrosis that is essential for multicellular organism development and immune response. The members of the GSDM superfamily (GSDMA, GSDMB, GSDMC, GSDMD, DFNA5 and DFNB59) share a similar pore-forming domain in their crystal structure, which is critical to execute pyroptosis induction (4-6). Recently, various studies have revealed the dysfunction and abnormal expression of the GSDM family in multiple human cancers $(7,8)$, implying the potential roles in tumorigenesis. Moreover, the clinical application of the GSDM family as diagnostic and prognostic factors for cancer patients is to be further elucidated in detail. Using high-resolution melting curve analysis, Lutkowska et al (7) found that a single nucleotide polymorphism (SNP) variant, rs8067378, significantly increased the expression of GSDMB, further leading to cervical carcinogenesis. Miguchi et al (8) demonstrated that GSDMC, functioning as a promising oncogene, promoted cell proliferation in colorectal cancer by depressing TGFBR2 (transforming growth factor $\beta$ receptor type II) activation. However, the potential functions and detailed mechanisms of the GSDM family in lung cancer are still unknown.

The present study was conducted to explore the detailed function and molecular mechanism of the GSDM family in LUAD biology. Using data from several bioinformatic platforms, the expression profiles of GSDM family members were evaluated in LUAD cases. GSDMC was found to be significantly upregulated in LUAD samples. Moreover, data from Kaplan-Meier curves showed that upregulated GSDMC acts as a negative prognostic indicator for LUAD patients. Mechanistically, based on data from The Cancer Genome 
Atlas (TCGA) database, the effect of DNA methylation value of $G S D M C$ on its expression and prognostic value were evaluated in LUAD patients. Meanwhile, the copy number alterations (CNAs) and mutation status of GSDMC DNA were also explored to further investigate GSDMC dysregulation in LUAD.

\section{Materials and methods}

Reanalysis of the acquisition data. The molecular profiles of GSDM family members in LUAD tissues and cell lines were identified from multiple bioinformatic platforms, such as Gene Expression Profiling Interactive Analysis (GEPIA), UALCAN and Oncomine. GEPIA is an online tool for gene expression profiling analyses in cancer and adjacent tissues (9). UALCAN platform is helpful for in-silico validation of tumor subgroup candidate biomarkers (10). Serving as a cancer microarray data-mining platform, Oncomine is valuable to characterize the gene expression signatures in multiple human cancer tissues and cells (11). The Cancer Cell Line Encyclopedia (CCLE) project provides public access for the systematic exploration of genetic and pharmacologic characterization in approximately 1,457 cell lines (12). The Human Protein Atlas Project, an international program, has been set up to draw a map of protein signatures on a cellular level (13). Using these bioinformatics data, the molecular profiles of the GSDM family were definitely characterized in LUAD patients.

Several bioinformatic tools, such as cBioPortal (14) and Kaplan-Meier Plotter (15), were used to reanalyze the clinical significance of GSDMC in LUAD cancer cases by linking TCGA clinical data to mRNA expression and DNA methylation values. In particular, using a TCGA-LUAD dataset (TCGA, Provisional) from cBioPortal, a retrospective study was performed to evaluate the association between GSDMC molecular profiles and clinical pathologic characteristics in LUAD patients. MethHC (16) and MEXPRESS (17) are two web databases that focus on the methylomes of human diseases, databases through which we confirmed the different methylation values between cancerous and noncancerous tissues. An important characteristic of MethHC and MEXPRESS is that all figures offer not just a visualization of the findings, but also a statistical analysis. The figures show the P-values and Pearson correlation coefficients for the comparison between the methylation value and expression level of the corresponding genes. The Kaplan-Meier algorithm was used to analyze the disease prognostic markers, such as first progression (FP), overall survival (OS) and postprogression survival (PPS). As for the Kaplan-Meier Plotter and DNA methylation analyses, all possible cut-off values between the lower and upper quartiles were analyzed by the algorithm automatically, and the best performing threshold was used as an optimal cut-off.

Subsequently, the copy number alterations (CNAs) and mutation status of GSDMC DNA were also downloaded from the cBioPortal web-server to re-analyze the potential roles of GSDMC in LUAD tumorigenesis.

In addition, we selected the abovementioned TCGA-LUAD dataset (TCGA, Provisional), which contained 517 RNA-sequenced samples, to analyze the coexpression genes of GSDMC. Using SangerBox tools, a Volcano Plot was drawn for a detailed visualization of differentially expressed genes
(DEGs), with the criteria of $\log _{2}$ (fold change) $\mid \geq 2$ and P-value $<0.05$. Moreover, the functional enrichment analysis of these DEGs, including Gene Ontology (GO) terms and Kyoto Encyclopedia of Genes and Genomes (KEGG) pathways, was performed using Database for Annotation, Visualization and Integration Discovery (DAVID) (18). The visualization of GO terms was showed by a web platform, WEB-based Gene SeT AnaLysis Toolkit (WebGestalt) (19).

All bioinformatic tools mentioned above are summarized in Table I.

Cell lines. The LUAD cell lines NCIH358 (ATCC CCL-5807), A549 (ATCC CCL-18), and their corresponding radio-resistant cells (NCIH358R and A549R) were kindly provided by the laboratory of Professor Deng (Winship Cancer Institute of Emory University, USA) $(20,21)$, and maintained in DMEM/ F-12 medium (Hyclone) containing 10\% fetal bovine serum (FBS) and $1 \%$ penicillin/gentamicin/streptomycin at $37^{\circ} \mathrm{C}$ with $5 \%$ carbon dioxide.

Real-time fluorescence quantitative PCR. As previously described (22), the total RNA was extracted from LUAD cells and used for cDNA synthesis. Real-time fluorescence quantitative PCR (qPCR) was performed using the CFX96 Touch Real-Time PCR Detection System (Bio-Rad) with Applied Biosystems Power SYBR Green PCR Master Mix (cat. no. 4367659, Thermo Fisher Scientific, Inc.). Amplification was performed in a three-step cycle procedure (denaturation at $95^{\circ} \mathrm{C}$ for $1 \mathrm{sec}$, ramp rate $20^{\circ} \mathrm{C} / \mathrm{sec}$; annealing at $60^{\circ} \mathrm{C}$ for $10 \mathrm{sec}$, ramp rate $20^{\circ} \mathrm{C} / \mathrm{sec}$; and extension at $72^{\circ} \mathrm{C}$ for $26 \mathrm{sec}$, ramp rate $2^{\circ} \mathrm{C} / \mathrm{sec}$ ) for 40 cycles. The involving primers were as follows: GSDMC forward, 5'-TCCGAAATGGTAGGCTAC TGT-3' and reverse, 5'-ATGAGGCATTGAAGAGGGTTG-3'; $\beta$-actin forward, 5'-CATGTACGTTGCTATCCAGGC-3' and reverse, 5'-CTCCTTAATGTCACGCACGAT-3'. PCR reaction with $\beta$-actin primers was used as an internal control. And the relative transcriptional level of GSDMC was normalized to $\beta$-actin. After the cycle threshold $(\mathrm{Cq})$ value (power amplification knee point) was achieved, we used the $2^{-\Delta \Delta \mathrm{Cq}}$ method to determine the relative expression levels (23). The specificity of amplification products was confirmed by the melting curve analysis. All experiments were repeated at least three times.

Statistical analyses. The differential expression of GSDMC between normal and LUAD samples was examined by the Student's t-test with SPSS 12.0 software (SPSS, Inc.). Wilcoxon's and Chi-square tests were performed to study associations between GSDMC molecular profiles and clinical pathologic characteristics. The significance of the difference between survival as determined by Kaplan-Meier curves was evaluated with the Logrank test. The correlation analysis was conducted by Pearson's correlation coefficient. The statistical differences were considered significant at a P-value of $<0.05$.

\section{Results}

Identification of upregulated GSDMC in LUAD tissues and cell lines. Using data from several bioinformatic tools, molecular profiles of the GSDM family members were evaluated between LUAD tissues and adjacent normal lung tissues. 
Table I. Bioinformatic tools used to analyze the roles of GSDMC in LUAD biology.

\begin{tabular}{ll}
\hline Database & \multicolumn{1}{c}{ URL } \\
\hline GEPIA & http://gepia.cancer-pku.cn/ \\
UALCAN & http://ualcan.path.uab.edu/index.html \\
Oncomine & https://www.oncomine.com/resource/login.html \\
CCLE & https://portals.broadinstitute.org/ccle \\
Human Protein Atlas project & http://www.proteinatlas.org/ \\
cBioPortal & http://www.cbioportal.org/ \\
Kaplan-Meier Plotter & http://kmplot.com/analysis/ \\
MethHC & http://methhc.mbc.nctu.edu.tw/php/index.php \\
MEXPRESS & http://mexpress.be/ \\
DAVID & https://david.ncifcrf.gov/ \\
WebGestalt & http://www.webgestalt.org/option.php
\end{tabular}

GSDMC, gasdermin C; LUAD, lung adenocarcinoma; GEPIA, Gene Expression Profiling Interactive Analysis; CCLE, Cancer Cell Line Encyclopedia; DAVID, Database for Annotation, Visualization and Integration Discovery.

The heatmap of data from UALCAN showed that GSDMA, $G S D M B, G S D M C$ and DFNB59 were all upregulated in LUAD compared with the levels in adjacent normal tissues (Fig. 1A). However, in a dataset (Selamat Lung) extracted from Oncomine, only GSDMB, GSDMC and DFNA5 were found to be significantly highly expressed in tumor tissues (Fig. 1B). Another heatmap result from GEPIA showed that GSDMA, GSDMB and GSDMC were significantly overexpressed in LUAD tissues (Fig. 1C). These findings collectively revealed that both $G S D M B$ and $G S D M C$ were consistently upregulated in LUAD patients. To verify these dysregulations, their expression was further detected in LUAD tissues using the IHC data from Human Protein Atlas project. As shown in Fig. 1D, the IHC results revealed that moderate GSDMB expression and negative GSDMC expression could be observed in normal lung tissues. In comparison, among the 8 cases of LUAD tissues examined for GSDMB staining, 6 cases had negative expression, while the remaining 2 cases had weak intensity. Conversely, among the 11 cases of LUAD tissues examined for GSDMC staining, 4 cases had strong or moderate staining, while the remaining 7 cases had negative expression. All these findings together demonstrated that $G S D M C$ is significantly upregulated in LUAD compared with adjacent normal tissues.

Next, the expression level of GSDMC was established in lung cancer cells. By exploring the CCLE database, a total of 75 LUAD cell lines with corresponding GSDMC expression values were finally selected. The heatmap data indicated that more than half of the bands were red, revealing the overexpressed GSDMC level in LUAD cells (Fig. 2A, Table SI). We also compared GSDMC expression levels in cancer cells with different metastatic abilities. The phenotypic differences in LUAD cells are summarized in Table SII. Expectedly, the expression level of GSDMC was significantly upregulated in metastatic LUAD cells (Fig. 2B). Then, the expression of $G S D M C$ was further determined in radio-resistant lung cancer cells. The qPCR analysis showed that GSDMC was obviously upregulated in radio-resistant LUAD cells (NCIH358R and A549R), compared with the parental cells (NCIH358 anc
A549) (Fig. 2C). Taken together, the above-mentioned data collectively demonstrated that overexpression of GSDMC acts as a promising oncogenic biomarker in LUAD.

Preserved GSDMC expression is an independent indicator for LUAD patients. Kaplan-Meier curves showed that the LUAD patients with low GSDMC expression had significantly better first progression (FP) $(\mathrm{P}=0.00001)$ and overall survival (OS) $(\mathrm{P}=0.0077)$ (Fig. 3A and $\mathrm{B})$. In addition, although patients with low GSDMC expression had a better post-progression survival (PPS), no statistical significance was achieved $(\mathrm{P}=0.22)$ (Fig. 3C). The associations between clinical pathological characteristics and GSDMC expression profiles are summarized in Table II. The findings showed that, compared with the patients with low GSDMC expression, the LUAD patients with high GSDMC expression had a significantly higher ratio of the KRAS mutation $(22 / 45,48.9 \%$ vs. $1 / 18,5.6 \% ; \mathrm{P}=0.001)$ and a longer history of cigarette smoking $(\mathrm{P}=0.012)$ (Table II).

DNA hypomethylation has a negative effect on GSDMC expression. The methylation status of GSDMC DNA was measured using an Illumina 450K Infinium methylation chip (Illumina, Inc.). The results from the MethHC web-tool showed that the GSDMC methylation level was significantly lower in LUAD tissues $(\mathrm{P}<0.05)$ (Fig. 4A). A significantly negative correlation was found between GSDMC expression and its DNA methylation status (Pearson's r=-0.136, $\mathrm{P}<0.001$ ) (Fig. 4B). Meanwhile, regression analysis from the MEXPRESS database also confirmed the negative relation between GSDMC expression and the methylation values of two CpG sites (cg05316065 and cg26073844) in LUAD cases (Pearson's $r=-0.175, P<0.001$ and Pearson's $\mathrm{r}=-0.141, \mathrm{P}<0.01$, respectively) (Fig. 4C). Based on its obvious significance, the $\mathrm{CpG}$ site, $\operatorname{cg} 05316065$, has been chosen to access the methylation status of GSDMC DNA on the LUAD patient clinical prognosis. Chi-square analysis showed that the patients with high methylation values of the CpG site (cg05316065) had a significantly higher Karnofsky performance score (KPS), an easy-to-use prognostic model for prognosis $(24,25)$. Moreover, the results suggested that the 
A

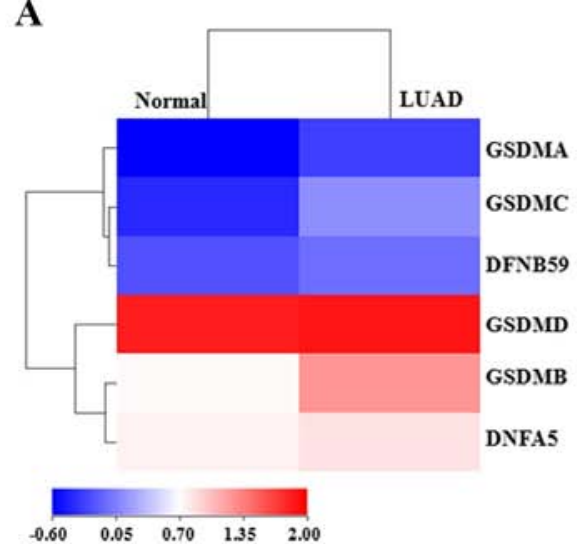

B

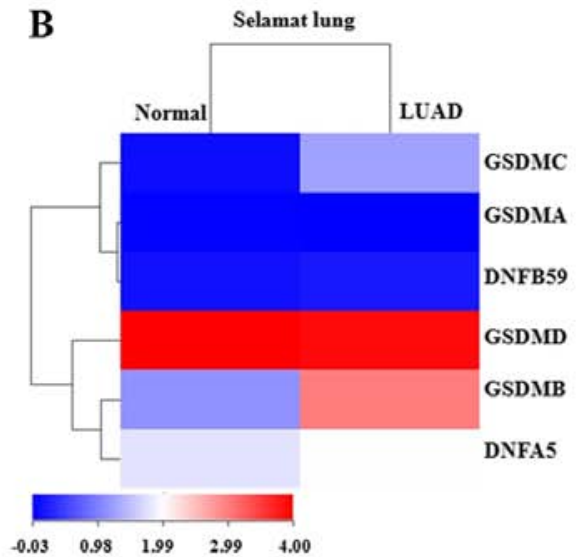

C

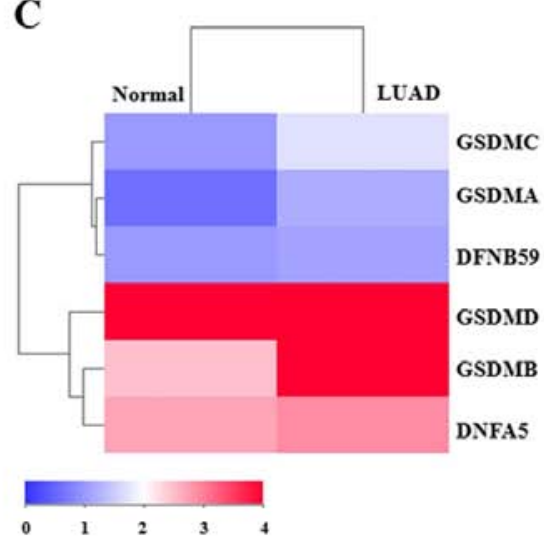

D

GSDMB

GSDMC

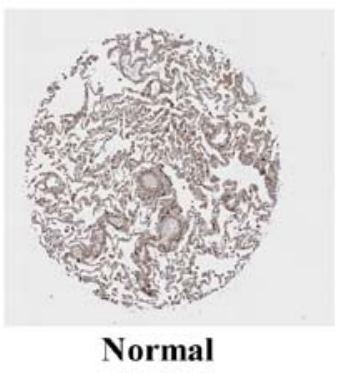

(x4)

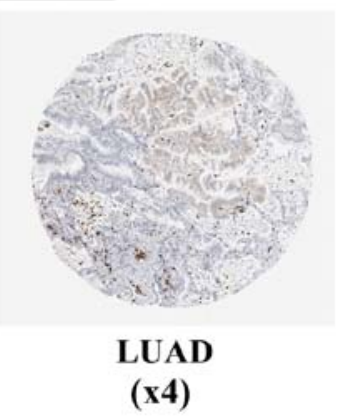

(x4)

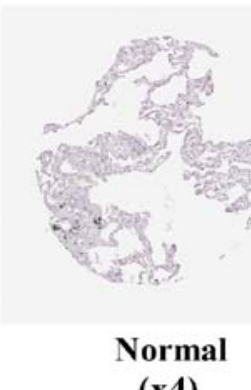

(x4)

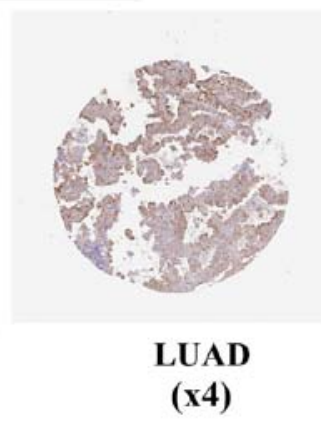

Figure 1. GSDMC is upregulated in LUAD tissues compared with normal lung tissues. (A-C) Heatmaps of the expression of GSDM superfamily genes (GSDMA, GSDMB, GSDMC, GSDMD, DFNA5 and DFNB59) between LUAD and paired normal samples. The results were generated by reanalysis of the data from UALCAN, Oncomine and GEPIA. (D) Representative GSDMB and GSDMC immunohistochemical (IHC) staining in normal lung and LUAD tissues. Image credit: The Human Protein Atlas Project. GSDM, gasdermin; LUAD, lung adenocarcinoma; UALCAN, University of Alabama Cancer Database; GEPIA, Gene Expression Profiling Interactive Analysis.
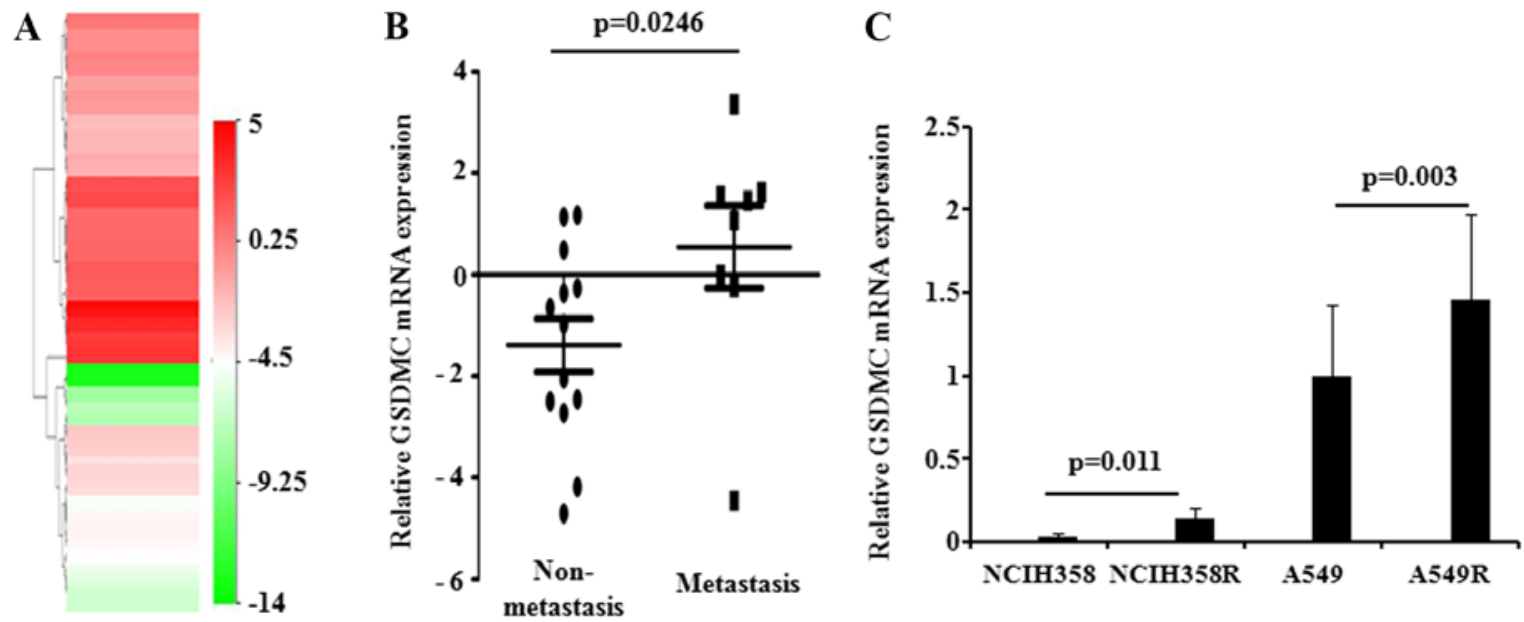

Figure 2. Identification of the overexpression of GSDMC in LUAD cells. (A) Heatmap for expression of GSDMC in LUAD cell lines. Red represents higher expression of GSDMC and blue represents lower expression. Each bar on the right part represents the expression of GSDMC in a lung cell line (contains 75 cell lines). (B) The expression levels of GSDMC in LUAD cell lines with different metastatic capability. (C) qPCR of the mRNA expression levels of GSDMC in radio-resistant LUAD cells. $\mathrm{P} \geq 0.05$ indicates a significant difference. GSDMC, gasdermin C; LUAD, lung adenocarcinoma.

cases with high methylation values of GSDMC had a longer OS and disease-free survival (DFS), although there were no significant differences (Table III).

Next, the relationship between GSDMC expression and its CNA/mutation status was also evaluated. We found that 581 samples among the 584 sequenced LUAD patients had CNAs measured, while only 45 samples (8\%) had an obvious copy number change (+2 high level amplification altered) (Fig. 5A). Additionally, the somatic mutation in GSDMC DNA was only found in 8 cases (1.4\%), with unknown significance (Fig. 5B). Thus, the CNA/mutation status did not influence GSDMC expression distinctly. 

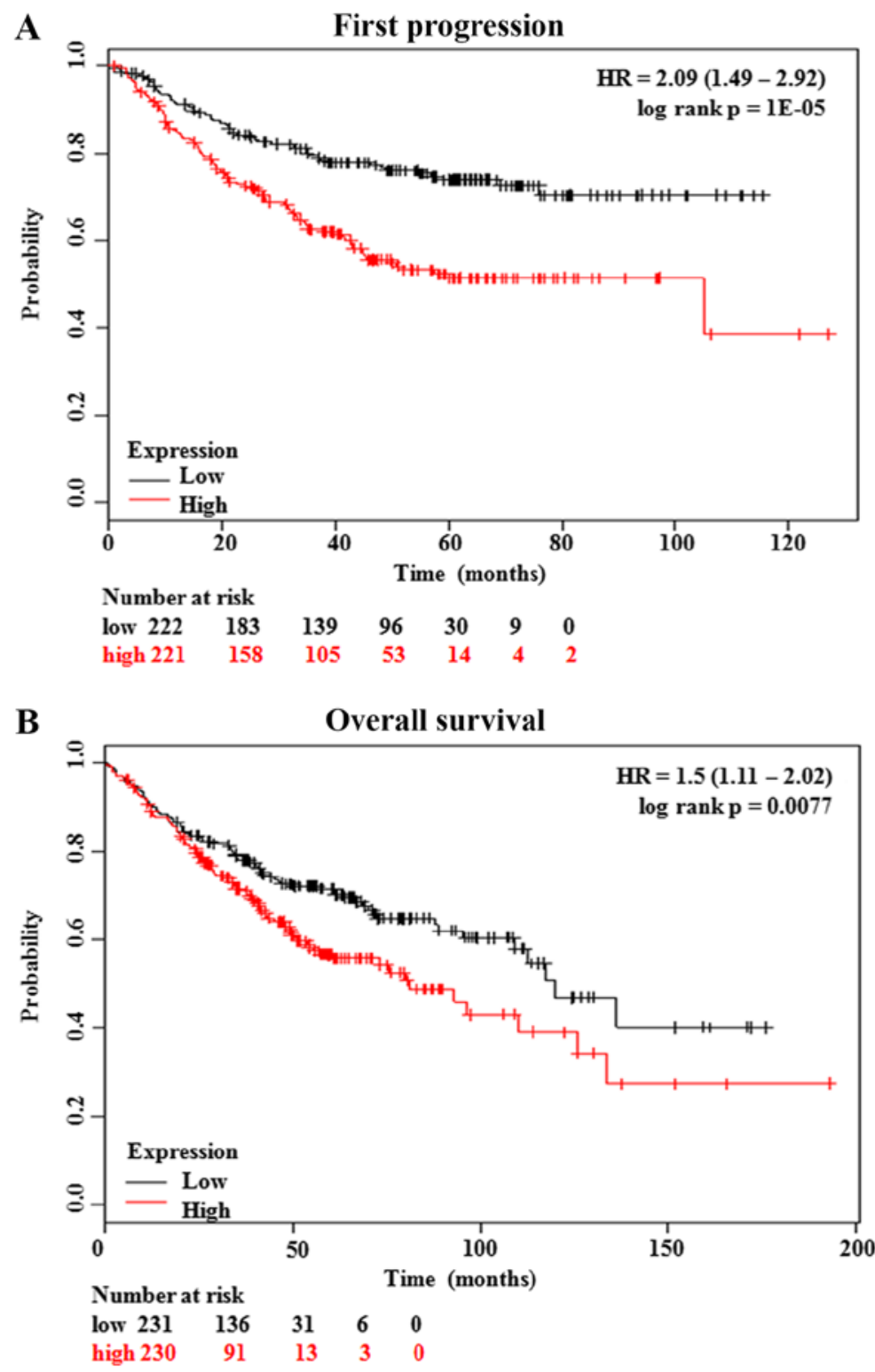

C

Post-progression survival

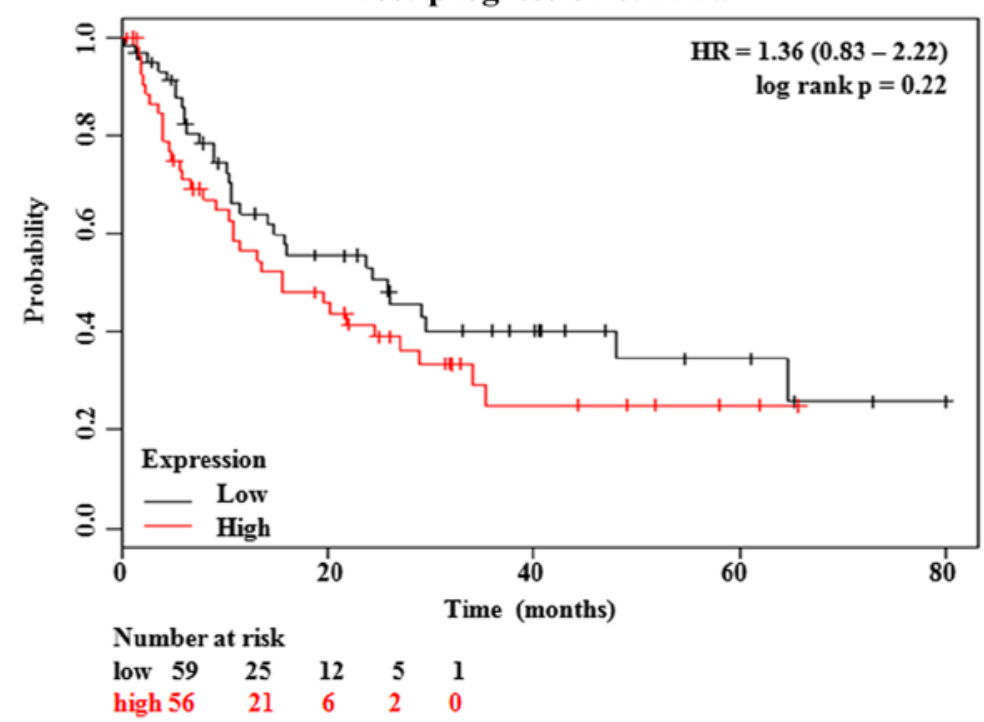

Figure 3. Kaplan-Meier curves of FP, OS and PPS in LUAD patients with different GSDMC expression. (A-C) The Kaplan-Meier Plotter platform was used to quickly confirm disease prognosis, such as FP, (A) OS (B) and PPS. (C) The Cancer Genome Atlas (TCGA) clinical data were used to categorize LUAD patients according to their expression levels of GSDMC. GSDMC, gasdermin C; LUAD, lung adenocarcinoma; FP, first progression; OS, overall survival; PPS, post-progression survival. 
Table II. Expression of GSDMC in the TCGA-LUAD datasets.

\begin{tabular}{|c|c|c|c|c|}
\hline Patient characteristics & No. of patients (\%) & High expression n (\%) & Low expression $\mathrm{n}(\%)$ & P-value \\
\hline $\operatorname{Sex}(N=515)$ & & & & 0.389 \\
\hline Male & $240(46.6)$ & $158(48.0)$ & $82(44.1)$ & \\
\hline Female & $275(53.4)$ & $171(52.0)$ & $104(55.9)$ & \\
\hline Lymph node stage $(\mathrm{N}=514)$ & & & & 0.326 \\
\hline N0 & $332(64.6)$ & $215(65.5)$ & $117(62.9)$ & \\
\hline N1 & $95(18.5)$ & $63(19.2)$ & $32(17.2)$ & \\
\hline $\mathrm{N} 2$ & $74(14.4)$ & $44(13.4)$ & $30(16.1)$ & \\
\hline $\mathrm{N} 3$ & $2(0.4)$ & $0(0.0)$ & $2(1.1)$ & \\
\hline NX & $11(2.1)$ & $6(1.8)$ & $5(2.7)$ & \\
\hline Metastasis stage $(\mathrm{N}=511)$ & & & & 0.922 \\
\hline M0 & $345(67.5)$ & $222(68.1)$ & $123(66.5)$ & \\
\hline M1 & $25(4.9)$ & $16(4.9)$ & $9(4.9)$ & \\
\hline MX & $141(27.6)$ & $88(27.0)$ & $53(28.6)$ & \\
\hline Tumor stage $(\mathrm{N}=515)$ & & & & 0.675 \\
\hline $\mathrm{T} 1$ & $168(32.6)$ & $110(33.4)$ & $58(31.2)$ & \\
\hline $\mathrm{T} 2$ & $278(54.0)$ & $175(53.2)$ & $103(55.4)$ & \\
\hline $\mathrm{T} 3$ & $47(9.1)$ & $29(8.8)$ & $18(9.7)$ & \\
\hline $\mathrm{T} 4$ & $19(3.7)$ & $14(4.3)$ & $5(2.7)$ & \\
\hline $\mathrm{TX}$ & $3(0.6)$ & $1(0.3)$ & $2(1.0)$ & \\
\hline Stage $(N=507)$ & & & & 0.808 \\
\hline I & $276(54.4)$ & $175(54.3)$ & $101(54.6)$ & \\
\hline II & $121(23.9)$ & $80(24.8)$ & $41(22.2)$ & \\
\hline III & $84(16.6)$ & $50(15.5)$ & $34(18.4)$ & \\
\hline IV & $26(5.1)$ & $17(5.3)$ & $9(4.9)$ & \\
\hline Race $(\mathrm{N}=448)$ & & & & 0.145 \\
\hline Asian & $8(1.8)$ & $3(1.1)$ & $5(3.0)$ & \\
\hline Black & $53(11.8)$ & $29(10.4)$ & $24(14.2)$ & \\
\hline Caucasian & $387(86.4)$ & $247(88.5)$ & $140(82.8)$ & \\
\hline Age $(N=496)$ in years & & & & 0.558 \\
\hline $20-40$ & $2(0.4)$ & $2(0.6)$ & $0(0.0)$ & \\
\hline $40-60$ & $134(27.0)$ & $81(25.6)$ & $53(29.4)$ & \\
\hline $60-80$ & $329(66.3)$ & $211(66.8)$ & $118(65.6)$ & \\
\hline$\geq 80$ & $31(6.3)$ & $22(7.0)$ & $9(5.0)$ & \\
\hline$K R A S$ mutation $(\mathrm{N}=63)$ & & & & 0.001 \\
\hline No & $40(63.5)$ & $23(51.1)$ & $17(94.4)$ & \\
\hline Yes & $23(36.5)$ & $22(48.9)$ & $1(5.6)$ & \\
\hline KPS (N=99) & & & & 0.908 \\
\hline $0-70$ & $12(12.1)$ & $6(11.8)$ & $6(12.5)$ & \\
\hline 80 & $23(23.2)$ & $13(25.5)$ & $10(20.8)$ & \\
\hline 90 & $32(32.3)$ & $17(33.3)$ & $15(31.3)$ & \\
\hline 100 & $32(32.3)$ & $15(29.4)$ & $17(35.4)$ & \\
\hline Cigarette smoking history, pack year $(\mathrm{N}=349)$ & & & & 0.012 \\
\hline$\leq 5$ & $7(2.0)$ & $5(2.2)$ & $2(1.6)$ & \\
\hline $6-20$ & $51(14.6)$ & $34(15.0)$ & $17(13.8)$ & \\
\hline $21-40$ & $115(33.0)$ & $81(35.8)$ & $34(27.6)$ & \\
\hline $41-60$ & $106(30.4)$ & $67(29.6)$ & $39(31.7)$ & \\
\hline $61-80$ & $33(9.5)$ & $17(7.5)$ & $16(13.0)$ & \\
\hline $81-100$ & $17(4.9)$ & $15(6.6)$ & $2(1.6)$ & \\
\hline$>100$ & $20(5.7)$ & $7(3.1)$ & $13(10.6)$ & \\
\hline
\end{tabular}
presented in bold print indicate significant differences. 
A

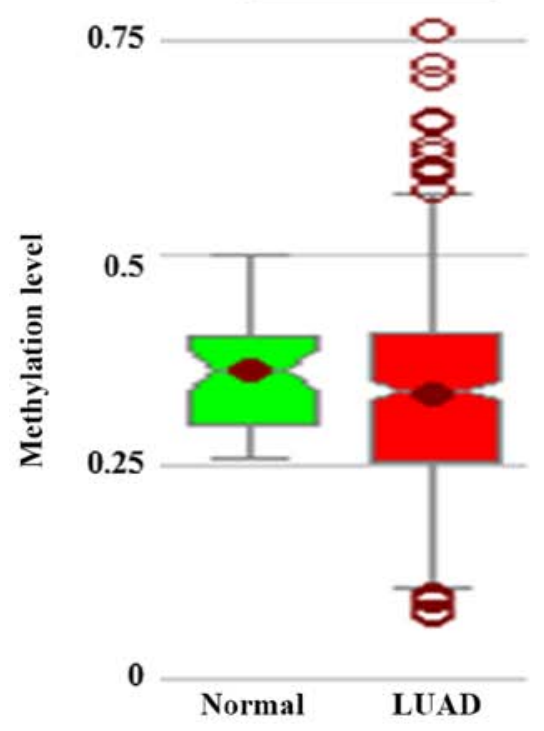

B

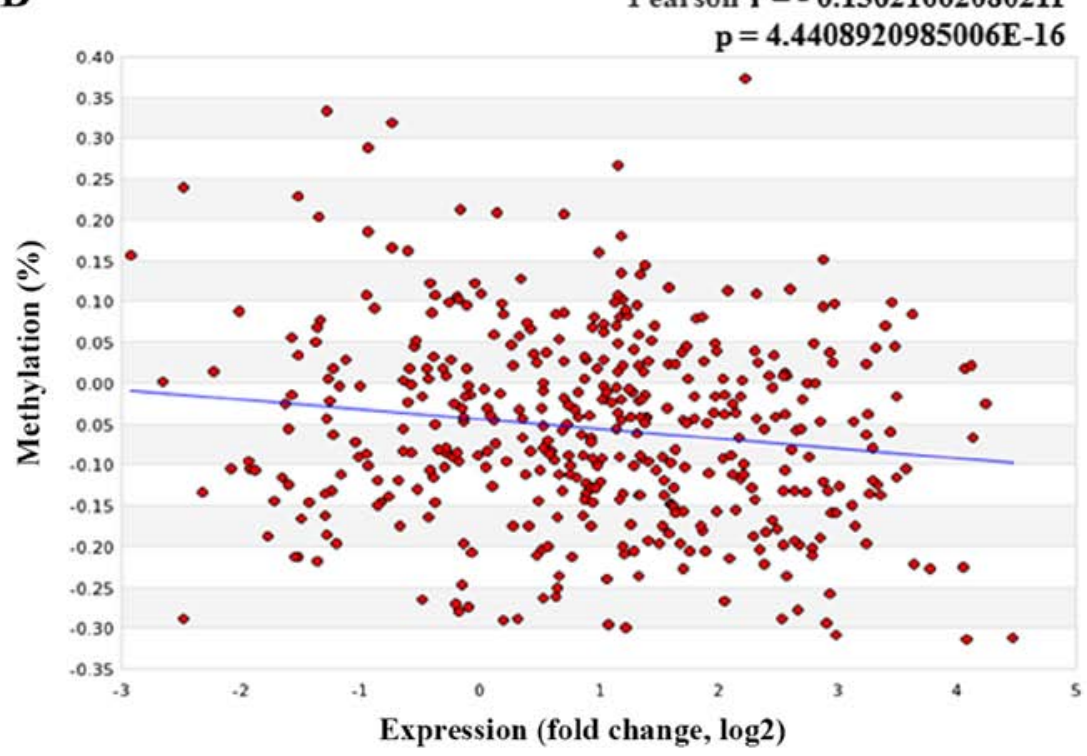

C

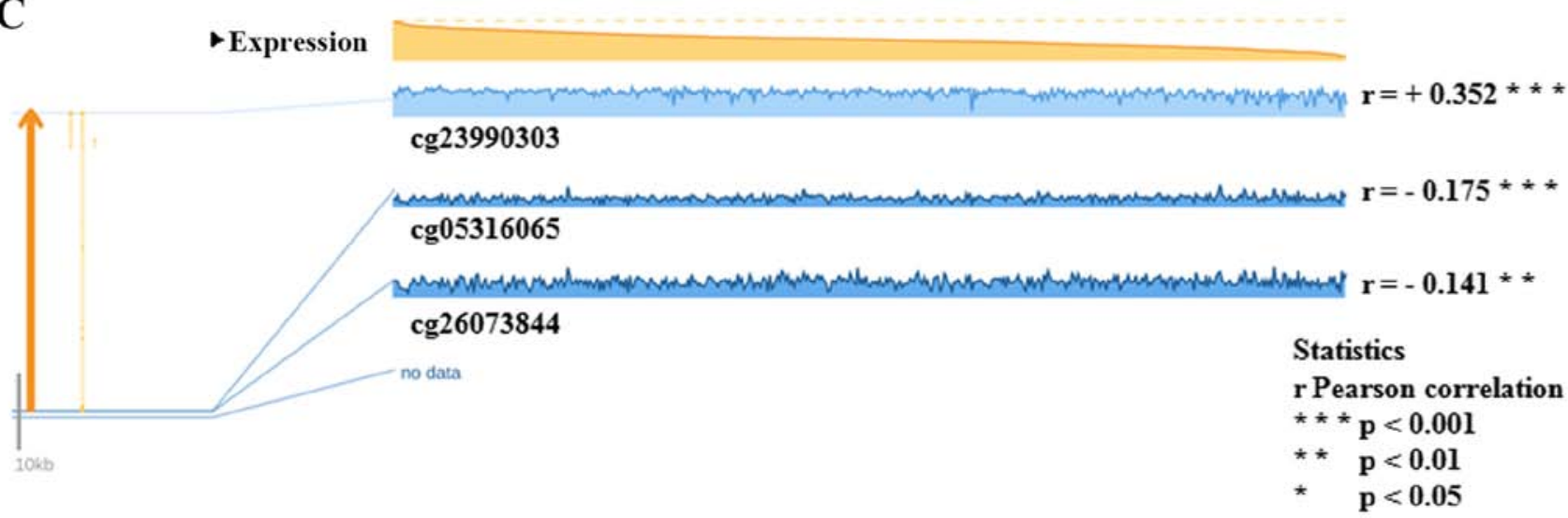

Figure 4. DNA hypomethylation may be a mechanism of the overexpression of GSDMC expression in LUAD. (A) Box-plot showing the methylation values between LUAD and normal lung tissues. (B) Regression analysis of the correlation between GSDMC expression and its DNA methylation in LUAD cases. (C) Regression analysis of the correlation between GSDMC expression and the DNA methylation of CpG sites in LUAD cases. GSDMC, gasdermin C; LUAD, lung adenocarcinoma.

Functional enrichment analysis of GSDMC-associated co-expression genes in LUAD patients. From a TCGA-LUAD dataset (TCGA, Provisional) of cBioPortal, 13,446 genes that were co-expressed with $G S D M C$ were identified in the LUAD samples. Then, using the criteria of $\log _{2}$ (fold change)l 32 and $\mathrm{P}$-value $<0.05,215$ genes were preliminarily validated as $G S D M C$-associated co-differentially expressed genes (co-DEGs) (Table SIII). These co-DEGs between the altered and unaltered GSDMC expression group are shown as a volcano plot (Fig. 6A). Next, GO and KEGG analyses were performed to clarify their biological function. The biological processes enrichment analysis showed that these co-DEGs were mainly correlated with biological regulation and metabolic processes. The cellular component of these co-DEGs was mainly enriched in the membrane and the nucleus. For molecular function analysis, these co-DEGs were enriched primarily in terms of protein binding (Fig. 6B). The KEGG pathway, annotated by the DAVID bioinformatics resource, indicated that these co-DEGs were mainly enriched in cell cycle regulation (hsa04110) (Fig. 6C).

\section{Discussion}

Research indicates that dysregulated GSDMC is associated with multiple cancer biological behaviors. Watabe et al (26) suggested that GSDMC overexpression promotes cell metastasis in B16 melanoma cells. In colorectal cancer cells, enforced GSDMC expression was found to enhance cell proliferation and tumorigenesis by suppressing TGFBR 2 activity (8). However, in gastric cancer cells, GSDMC was found to exert obvious cell-growth inhibition activity, functioning as a potential tumor suppressor (27). These findings collectively demonstrate that the detailed functional roles of GSDMC are tissue-specific.

By using several public databases, our present study presented the expression profiles of GSDM superfamily members in LUAD, which revealed that GSDMC is obviously upregulated in LUAD tissues and cell lines. We also found that the LUAD patients with higher GSDMC expression presented with significant KRAS mutation status. Studies have revealed that patient prognosis after LUAD diagnosis is usually poor, and current treatment strategies derived from primary LUAD seem to be 
A Profiled in Putative copy-number alterations from GISTIC Profiled in mRNA Expression z-Scores (microarray)

GSDMC

$8 \%$

Genetic Alteration | Amplification | mRNA vpregulation || No alterations "Not profiled

Profiled in Putative copy-number \| Yes - No alterations from GISTIC

Profiled in mRNA Expression

z-Scores (microarray)

IVes $-\mathrm{N}_{0}$

B Profiled in Mutations

Profiled in mRNA Expression z-Scores (microarray)

GSDMC

$1.4 \%$

Genetic Alteration

|| Missesse Sutation (untrom siguificance) || mRNA Upregulation || No alterations - Not profiled

Profiled in Mutations

IYes - No

Profiled in mRNA Expression

z-Scores (microarray)

IYes - No

Figure 5. GSDMC DNA CNAs and mutations. (A) Heatmap showing the correlation between GSDMC expression and its DNA CNAs. The data indicated that GSDMC expression was altered only in $8 \%$ of cases with CNAs (+2 high level amplification altered). (B) Heatmap showing the correlation between GSDMC expression and its DNA mutations in LUAD patients. GSDMC, gasdermin C; LUAD, lung adenocarcinoma; CNAs, copy number alterations.

A

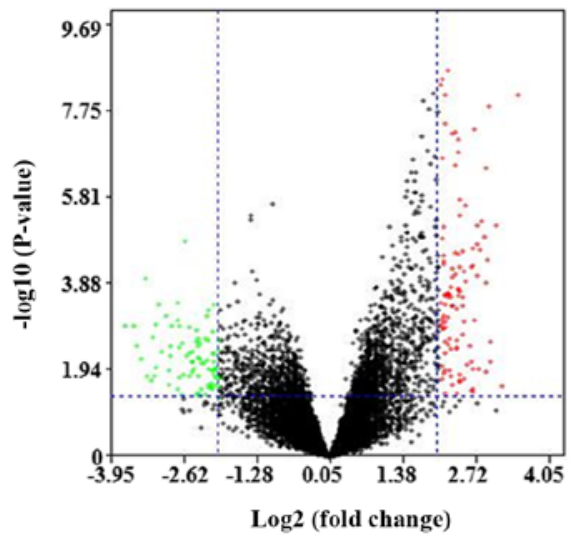

C

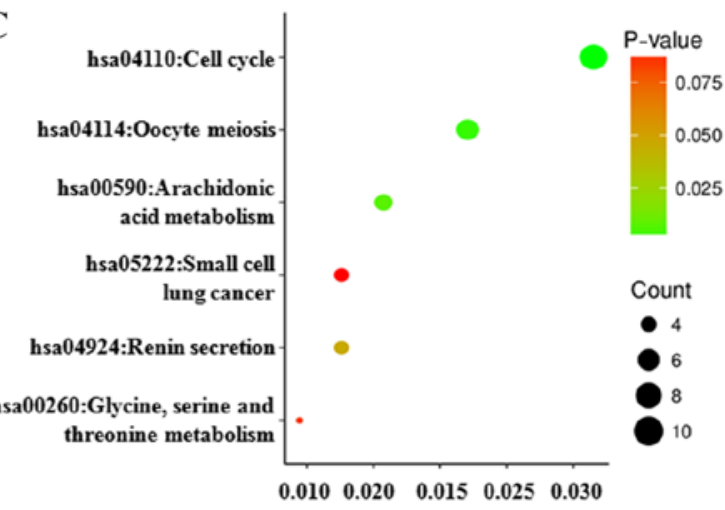

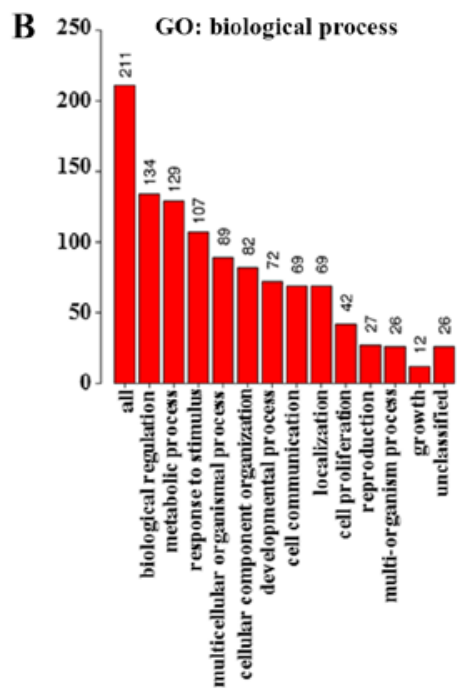
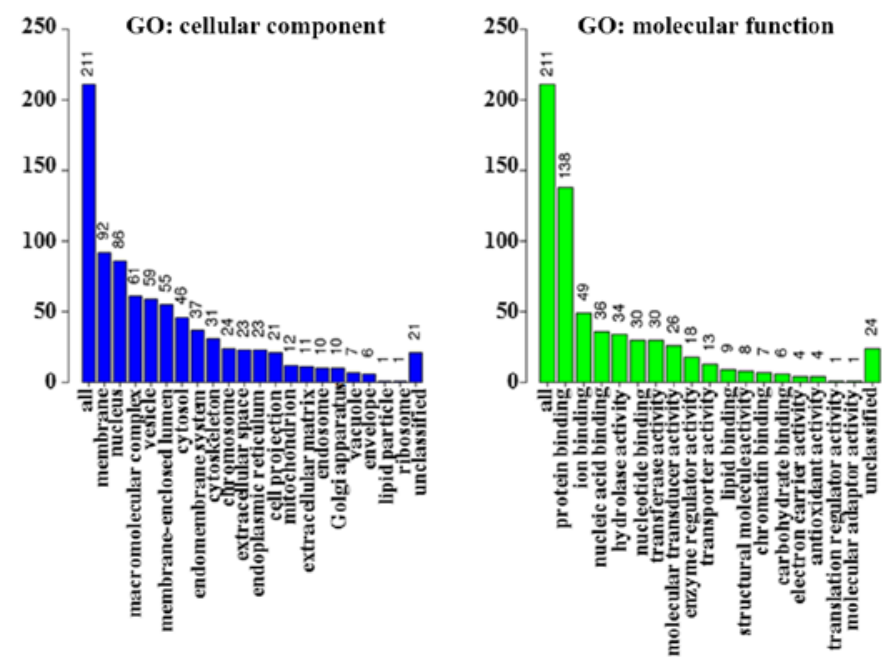

Figure 6. Function of enrichment analysis concerning GSDMC-related genes in LUAD patients. (A) A volcano plot showing GSDMC-associated co-expressed genes between the altered and unaltered GSDMC expression group in the selected TCGA-LUAD data (TCGA, Provisional). (B) The top significant GO biological process enrichments, including biological regulation and metabolic processes. The top dysregulated cellular component in LUAD is the membrane and nucleus. The GO enrichment analysis of molecular function is primarily enriched in terms of protein binding. (C) KEGG pathway analyses of GSDMC-related co-DEGs in the selected TCGA-LUAD data (TCGA, Provisional). GSDMC, gasdermin C; LUAD, lung adenocarcinoma; TCGA, The Cancer Genome Atlas; GO, Gene Ontology; KEGG, Kyoto Encyclopedia of Genes and Genomes. 
Table III. The cg05316065 methylation status of GSDMC in the TCGA-LUAD datasets.

\begin{tabular}{|c|c|c|c|c|}
\hline \multirow[b]{2}{*}{ Patient characteristics } & \multicolumn{3}{|c|}{ cg05316065 methylation value } & \multirow[b]{2}{*}{ P-value } \\
\hline & Total no. of patients (\%) & High methylation & Low methylation & \\
\hline $\operatorname{Sex}(N=447)$ & & & & 0.008 \\
\hline Male & $208(46.5)$ & $118(52.9)$ & $90(40.2)$ & \\
\hline Female & $239(53.5)$ & $105(47.1)$ & $134(59.8)$ & \\
\hline Lymph node stage $(\mathrm{N}=445)$ & & & & 0.317 \\
\hline No & $290(65.2)$ & $149(67.1)$ & $141(63.2)$ & \\
\hline N1 & $82(18.4)$ & $35(15.8)$ & $47(21.1)$ & \\
\hline $\mathrm{N} 2$ & $64(14.4)$ & $35(15.8)$ & $29(13.0)$ & \\
\hline NX & $9(2.0)$ & $3(1.4)$ & $6(2.7)$ & \\
\hline Metastasis stage $(\mathrm{N}=443)$ & & & & 0.701 \\
\hline M0 & $292(65.9)$ & $143(64.4)$ & $149(67.4)$ & \\
\hline M1 & $19(4.3)$ & $11(5.0)$ & $8(3.6)$ & \\
\hline MX & $132(29.8)$ & $68(30.6)$ & $64(29.0)$ & \\
\hline Tumor stage $(\mathrm{N}=447)$ & & & & 0.101 \\
\hline $\mathrm{T} 1$ & $150(33.6)$ & $67(30.0)$ & $83(37.1)$ & \\
\hline $\mathrm{T} 2$ & $239(53.5)$ & $119(53.4)$ & $120(53.6)$ & \\
\hline $\mathrm{T} 3$ & $39(8.7)$ & $25(11.2)$ & $14(6.3)$ & \\
\hline $\mathrm{T} 4$ & $16(3.6)$ & $11(4.9)$ & $5(2.2)$ & \\
\hline $\mathrm{TX}$ & $3(0.7)$ & $1(0.4)$ & $2(0.9)$ & \\
\hline Stage $(N=442)$ & & & & 0.591 \\
\hline I & $241(54.5)$ & $118(53.2)$ & $123(55.9)$ & \\
\hline II & $109(24.7)$ & $52(23.4)$ & $57(25.9)$ & \\
\hline III & $72(16.3)$ & $41(18.5)$ & $31(14.1)$ & \\
\hline IV & $20(4.5)$ & $11(5.0)$ & $9(4.1)$ & \\
\hline Race $(\mathrm{N}=399)$ & & & & 0.813 \\
\hline Asian & $6(1.5)$ & $4(2.0)$ & $2(1.0)$ & \\
\hline Black & $40(10.0)$ & $20(10.0)$ & $20(10.1)$ & \\
\hline Caucasian & $353(88.5)$ & $176(88.0)$ & $177(88.9)$ & \\
\hline Age $(N=428)$ in years & & & & 0.522 \\
\hline $20-40$ & $0(0.0)$ & $0(0.0)$ & $0(0.0)$ & \\
\hline $40-60$ & $119(27.8)$ & $59(27.2)$ & $60(28.4)$ & \\
\hline $60-80$ & $280(65.4)$ & $146(67.3)$ & $134(63.5)$ & \\
\hline$\geq 80$ & $29(6.8)$ & $12(5.5)$ & $17(8.1)$ & \\
\hline$K R A S$ mutation $(\mathrm{N}=61)$ & & & & 0.979 \\
\hline No & $38(62.3)$ & $15(62.5)$ & $23(62.2)$ & \\
\hline Yes & $23(37.7)$ & $9(37.5)$ & $14(37.7)$ & \\
\hline KPS (N=83) & & & & 0.044 \\
\hline $0-70$ & $11(13.3)$ & $4(8.9)$ & $7(18.4)$ & \\
\hline 80 & $19(22.9)$ & $11(24.4)$ & $8(21.1)$ & \\
\hline 90 & $24(28.9)$ & $9(20.0)$ & $15(39.4)$ & \\
\hline 100 & $29(34.9)$ & $21(46.7)$ & $8(21.1)$ & \\
\hline Median DFS (95\% CI) & $33.44(24.70-42.18)$ & $33.44(24.73-42.14)$ & $30.39(15.77-45.01)$ & 0.823 \\
\hline Median OS (95\% CI) & $49.24(41.29-57.19)$ & $50.3(38.09-62.51)$ & $49.21(42.42-56.00)$ & 0.921 \\
\hline
\end{tabular}

GSDMC, gasdermin C; LUAD, lung adenocarcinoma; KPS, Karnofsky performance scores; TCGA, The Cancer Genome Atlas; DFS, disease-free survival; OS, overall survival; CI, confidence interval. P-values presented in bold print indicate significant differences.

largely inefficacious (28). Next, we detected the potential influence of GSDMC expression on the prognostic markers of LUAD patients, in terms of first progression (FP), overall survival (OS) and post-progression survival (PPS). Kaplan-Meier curves showed significantly better FP and OS in the lower GSDMC staining group, but no statistical difference in PPS cases with different GSDMC expression. These unexpected findings may be due to the small sample size and may need to be further examined in future studies. The following analysis of the association between GSDMC profiles and clinicopathological parameters 
further support that preserved GSDMC expression could act as an independent prognostic indicator for LUAD patients.

Research has revealed that epigenetic alterations (such as DNA hypomethylation) and genetic mutations have key functions in modulating transcription of important oncogenes in several types of human carcinomas, including LUAD (29-33). For example, Upchurch et al (34) found that aberrant promoter hypomethylation frequently affected the expression levels of several oncogenes in hematologic malignancies. Preferentially expressed phosphoribosylaminoimidazole carboxylase (PAICS) has been confirmed as a major tumor promoter in LUAD, which is frequently activated by promoter DNA hypomethylation (33). Cancer/testis antigen 45 (CT45) has been confirmed to be associated with disease progression of epithelial ovarian cancer, which is frequently activated by promoter DNA hypomethylation (35). Unexpectedly, the CNAs in CT45 DNA did not obviously influence its expression (35). In addition, Wang et al (29) demonstrated that tumor-suppressive activity by adenosine deaminase RNA-specific Bl (ADARB1) overexpression is a consequence of DNA demethylation signaling. Inactivation of DNA methylation using DNA methyltransferase inhibitors markedly enhanced the ADARB1-mediation metastatic inhibition of LUAD cells. Here, using the data from an Illumina 450K Infinium methylation chip, we identified that GSDMC was hypomethylated in LUAD samples compared with that in normal lung samples $(\mathrm{P}<0.05)$ (Fig. 4A). In addition, a significant negative correlation was found between GSDMC expression and its DNA methylation status in all LUAD cases (Pearson's $r=-0.136$ ). Meanwhile, the results from the MEXPRESS database confirmed that the expression of $G S D M C$ was negatively correlated to the methylation values of two CpG sites (cg05316065 and cg26073844) in its promoter region (Pearson's $r=-0.175$ and -0.141 , respectively). Although this is the first study to identify these two hypomethylated CpG sites in GSDMC and to demonstrate several advances in indicating their functions in LUAD tumorigenesis, many of the functional mechanisms remain poorly understood; particularly in terms of their roles in cancer metastasis, risk index and therapeutic response. Moreover, in this report, the LUAD patients with longer OS also had higher GSDMC methylation values. In comparison, GSDMC DNA CNAs and somatic mutation were not frequent events, and might not obviously affect the transcriptional level. Thus, these experiments confirmed the important functions of DNA hypomethylation for the overexpression of GSDMC in LUAD biological processes.

Alterations in cell cycle regulation have been well-described to date in human carcinogenesis as they are known to play pivotal roles in cell differentiation and growth. Identifying the changes in cell cycle checkpoints is essential to the regulation of cell cycle processes and the maintenance of genome integrity. Abnormal activation of oncogenes or various cancer therapeutic strategies could evoke cell cycle arrest in malignant cells, thereby interfering with tumor formation or progression (36). Moreover, studies have shown that tight temporal control of cell cycle checkpoints has a potential application in clinical practice. The pharmacological inhibitors that target cell cycle checkpoints have the potential to provoke the synthetic lethality of anticancer therapeutics and disrupt the cancerous phenotype (37). Esophageal squamous cell carcinoma cells treated with chemotherapeutic agents have impaired cell cycle progression and DNA damage repair by promoting the accumulation of GSDME around the cytoplasm (38). In our study, the detailed molecular interactions between GSDMC and its co-expressed genes were not well-illuminated; however, the GO and KEGG analyses that were constructed identified the function of GSDMC to some extent. Using the gene-annotation enrichment analysis, the main functional pathway of the GSDMC co-expressed genes was confirmed to be cell cycle regulation. However, no relevant studies have demonstrated the roles and mechanisms of GSDMC in cell cycle modulation. Therefore, further research is required to clarify the detailed functions of GSDMC in LUAD biology.

In conclusion, collectively, our present study revealed that $G S D M C$ is significantly overexpressed in LUAD tissues and cell lines. We also found that upregulated GSDMC expression could act as an independent predictor for the poor prognosis of LUAD patients. Mechanistically, GSDMC expression may be modulated by DNA hypomethylation in LUAD cells.

\section{Acknowledgements}

Not applicable.

\section{Funding}

The present study was supported by grants from the Natural Science Foundation of Hunan Province, China (2019JJ50932), the National Natural Science Foundation of China (81803035, 81703036, 81572946), China Postdoctoral Science Foundation (2017M610510) and the Youth Fund of Xiangya Hospital (2017Q17).

\section{Availability of data and materials}

All data generated or analyzed during this study are included in this published article.

\section{Authors' contributions}

Literature search was carried out by ZX, JW, ZG and YY. Data collection was performed by XC, XW, SZ and LQ. The study design was the responsibility of YY and ZX. Analysis of the data was carried out by XY, CO and WL, and manuscript preparation was completed by $\mathrm{YY}$ and $\mathrm{ZX}$. Review of the manuscript was undertaken by YY and ZX. All authors read and approved the manuscript and agree to be accountable for all aspects of the research in ensuring that the accuracy or integrity of any part of the work are appropriately investigated and resolved.

\section{Ethics approval and consent to participate}

Not applicable.

\section{Patient consent for publication}

Not applicable.

\section{Competing interests}

The authors declare that they have no competing interests. 


\section{References}

1. Miller KD, Siegel RL, Lin CC, Mariotto AB, Kramer JL, Rowland JH, Stein KD, Alteri R and Jemal A: Cancer treatment and survivorship statistics, 2016. CA Cancer J Clin 66: 271-289, 2016.

2. Yan Y, Su W, Zeng S, Qian L, Chen X, Wei J, Chen N, Gong Z and $\mathrm{Xu} \mathrm{Z}$ : Effect and mechanism of tanshinone I on the radiosensitivity of lung cancer cells. Mol Pharm 15: 4843-4853, 2018.

3. Jordan EJ, Kim HR, Arcila ME, Barron D, Chakravarty D, Gao J, Chang MT, Ni A, Kundra R, Jonsson P, et al: Prospective comprehensive molecular characterization of lung adenocarcinomas for efficient patient matching to approved and emerging therapies. Cancer Discov 7: 596-609, 2017.

4. Kovacs SB and Miao EA: Gasdermins: Effectors of pyroptosis. Trends Cell Biol 27: 673-684, 2017.

5. Shi J, Gao W and Shao F: Pyroptosis: Gasdermin-mediated programmed necrotic cell death. Trends Biochem Sci 42: 245-254, 2017.

6. Yuan YY, Xie KX, Wang SL and Yuan LW: Inflammatory caspase-related pyroptosis: Mechanism, regulation and therapeutic potential for inflammatory bowel disease. Gastroenterol Rep (Oxf) 6: 167-176, 2018

7. Lutkowska A, Roszak A, Lianeri M, Sowinska A, Sotiri E and Jagodzinski PP: Analysis of rs 8067378 polymorphism in the risk of uterine cervical cancer from a polish population and its impact on gasdermin B expression. Mol Diagn Ther 21: 199-207, 2017.

8. Miguchi M, Hinoi T, Shimomura M, Adachi T, Saito Y, Niitsu H, Kochi M, Sada H, Sotomaru Y, Ikenoue T, et al: Gasdermin C is upregulated by inactivation of transforming growth factor $\beta$ receptor type II in the presence of mutated apc, promoting colorectal cancer proliferation. PloS One 11: e0166422, 2016.

9. Tang Z, Li C, Kang B, Gao G, Li C and Zhang Z: GEPIA: A web server for cancer and normal gene expression profiling and interactive analyses. Nucleic Acids Res 45: W98-W102, 2017

10. Chandrashekar DS, Bashel B, Balasubramanya SAH, Creighton CJ, Ponce-Rodriguez I, Chakravarthi BVSK and Varambally S: UALCAN: A portal for facilitating tumor subgroup gene expression and survival analyses. Neoplasia 19: 649-658, 2017.

11. Rhodes DR, Kalyana-Sundaram S, Mahavisno V, Varambally R, Yu J, Briggs BB, Barrette TR, Anstet MJ, Kincead-Beal C, Kulkarni P, et al: Oncomine 3.0: Genes, pathways, and networks in a collection of 18,000 cancer gene expression profiles. Neoplasia 9: 166-180, 2007.

12. Barretina J,Caponigro G,Stransky N, Venkatesan K, Margolin AA, Kim S, Wilson CJ, Lehár J, Kryukov GV, Sonkin D, et al: The cancer cell line encyclopedia enables predictive modelling of anticancer drug sensitivity. Nature 483: 603-607, 2012.

13. Uhlen M, Zhang C, Lee S, Sjöstedt E, Fagerberg L, Bidkhori G, Benfeitas R, Arif M, Liu Z, Edfors F, et al: A pathology atlas of the human cancer transcriptome. Science 357: eaan2507, 2017.

14. Gao J, Aksoy BA, Dogrusoz U, Dresdner G, Gross B, Sumer SO, Sun Y, Jacobsen A, Sinha R, Larsson E, et al: Integrative analysis of complex cancer genomics and clinical profiles using the cBioPortal. Sci Signal 6: pl1, 2013.

15. Gyorffy B, Surowiak P, Budczies J and Lanczky A: Online survival analysis software to assess the prognostic value of biomarkers using transcriptomic data in non-small-cell lung cancer. PloS One 8: e82241, 2013.

16. Huang WY, Hsu SD, Huang HY, Sun YM, Chou CH, Weng SL and Huang HD: MethHC: A database of DNA methylation and gene expression in human cancer. Nucleic Acids Res 43: D856-D861, 2015.

17. Koch A, De Meyer T, Jeschke J and Van Criekinge W: MEXPRESS: Visualizing expression, DNA methylation and clinical TCGA data. BMC Genomics 16: 636, 2015.

18. Huang DW, Sherman BT, Tan Q, Kir J, Liu D, Bryant D, Guo Y, Stephens R, Baseler MW, Lane HC and Lempicki RA: DAVID Bioinformatics Resources: Expanded annotation database and novel algorithms to better extract biology from large gene lists. Nucleic Acids Res 35: W169-W175, 2007.

19. Wang J, Duncan D, Shi Z and Zhang B: WEB-based GEne SeT analysis toolkit (WebGestalt): Update 2013. Nucleic Acids Res 41: W77-W83, 2013.

20. Xu Z, Yan Y, Xiao L, Dai S, Zeng S, Qian L, Wang L, Yang X, Xiao $Y$ and Gong $Z$ : Radiosensitizing effect of diosmetin on radioresistant lung cancer cells via Akt signaling pathway. PloS One 12: e0175977, 2017.
21. You S, Li R, Park D, Xie M, Sica GL, Cao Y, Xiao ZQ and Deng X: Disruption of STAT3 by niclosamide reverses radioresistance of human lung cancer. Mol Cancer Ther 13: 606-616, 2014.

22. Dai S, Yan Y, Xu Z, Zeng S, Qian L, Huo L, Li X, Sun L and Gong Z: SCD1 confers temozolomide resistance to human glioma cells via the Akt/GSK3 $/ \beta$-catenin signaling axis. Front Pharmacol 8: 960, 2017.

23. Livak KJ and Schmittgen TD: Analysis of relative gene expression data using real-time quantitative PCR and the 2(-Delta Delta C(T)) method. Methods 25: 402-408, 2001.

24. Ouyang Z, Peng D and Dhakal DP: Risk factors for hematological toxicity of chemotherapy for bone and soft tissue sarcoma. Oncol Lett 5: 1736-1740, 2013.

25. TandonP,ReddyKR,O'LearyJG,Garcia-TsaoG,Abraldes JG, WongF, Biggins SW, Maliakkal B, Fallon MB, Subramanian RM, et al: A Karnofsky performance status-based score predicts death after hospital discharge in patients with cirrhosis. Hepatology 65: 217-224, 2017.

26. Watabe K, Ito A, Asada H, Endo Y, Kobayashi T, Nakamoto K, Itami S, Takao S, Shinomura Y, Aikou T, et al: Structure, expression and chromosome mapping of MLZE, a novel gene which is preferentially expressed in metastatic melanoma cells. Jap J Cancer Res 92: 140-151, 2001.

27. Saeki N, Usui T, Aoyagi K, Kim DH, Sato M, Mabuchi T, Yanagihara K, Ogawa K, Sakamoto H, Yoshida T and Sasaki H: Distinctive expression and function of four GSDM family genes (GSDMA-D) in normal and malignant upper gastrointestinal epithelium. Genes Chromosomes Cancer 48: 261-271, 2009.

28. Borczuk AC: Prognostic considerations of the new world health organization classification of lung adenocarcinoma. Eur Respir Rev 25: 364-371, 2016.

29. Wang X, Xu Z, Ren X, Chen X, Wei J, Lin W, Li Z, Ou C, Gong Z and Yan Y: Function of low ADARB1 expression in lung adenocarcinoma. PLoS One 14: e0222298, 2019.

30. Wang X, Xu Z, Chen X, Ren X, Wei J, Zhou S, Yang X, Zeng S, Qian L, Wu G, et al: A tropomyosin receptor kinase family protein, NTRK2 is a potential predictive biomarker for lung adenocarcinoma. PeerJ 7: e7125, 2019.

31. Yang X, Xu ZJ, Chen X, Zeng SS, Qian L, Wei J, Peng M, Wang X, Liu WL, Ma HY, et al: Clinical value of preoperative methylated septin 9 in Chinese colorectal cancer patients. World J Gastroenterol 25: 2099-2109, 2019.

32. Chen X, Xu Z, Zeng S, Wang X, Liu W, Qian L, Wei J, Yang X, Shen Q, Gong Z and Yan Y: SIRT5 downregulation is associated with poor prognosis in glioblastoma. Cancer Biomark 24: 449-459, 2019

33. Zhou S, Yan Y, Chen X, Wang X, Zeng S, Qian L, Wei J, Yang X, Zhou Y, Gong Z and Xu Z: Roles of highly expressed PAICS in lung adenocarcinoma. Gene 692: 1-8, 2019.

34. Upchurch GM, Haney SL and Opavsky R: Aberrant promoter hypomethylation in CLL: Does it matter for disease development? Front Oncol 6: 182, 2016.

35. Zhang W, Barger CJ, Link PA, Mhawech-Fauceglia P, Miller A, Akers SN, Odunsi K and Karpf AR: DNA hypomethylation-mediated activation of Cancer/Testis Antigen 45 (CT45) genes is associated with disease progression and reduced survival in epithelial ovarian cancer. Epigenetics 10: 736-748, 2015.

36. Milanovic M, Yu Y and Schmitt CA: The senescence-stemness alliance-a cancer-hijacked regeneration principle. Trends Cell Biol 28: 1049-1061, 2018 .

37. Vymětalová L and Kryštof V: Potential clinical uses of CDK inhibitors: Lessons from synthetic lethality screens. Med Res Rev 35: 1156-1174, 2015.

38. Wu M, Wang Y, Yang D, Gong Y, Rao F, Liu R, Danna Y, Li J, Fan J, Chen J, et al: A PLK1 kinase inhibitor enhances the chemosensitivity of cisplatin by inducing pyroptosis in oesophageal squamous cell carcinoma. EBioMedicine 41: 244-255, 2019

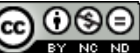

This work is licensed under a Creative Common Attribution-NonCommercial-NoDerivatives 4.0 International (CC BY-NC-ND 4.0) License. 